\title{
An Efficient Algorithm for Determination of the Optimum Base-Station Assignment in Cellular DS-CDMA Systems
}

\author{
Luis Mendo and José M. Hernando, Member, IEEE
}

\begin{abstract}
An algorithm is proposed that finds the optimum assignment of mobile users to base stations, and its associated transmission powers, in a cellular direct-sequence code-division multiple-access network, with a computational complexity that grows polynomially with the number of users and base stations. The algorithm detects infeasible situations and allows the inclusion of power constraints. Its performance is analyzed in terms of complexity and system capacity.
\end{abstract}

Index Terms-Code-division multiple access (CDMA), land mobile radio cellular systems, power control, user capacity.

\section{INTRODUCTION}

D IRECT-SEQUENCE code-division multiple access (DSCDMA) poses new challenges in the design and operation of cellular communication networks. Especially important is the optimum assignment of user equipment to base stations. The interference-limited feature of CDMA cellular networks implies that such assignment should not be done on a minimum attenuation basis (as in current frequency-division multiple access (FDMA) or time-division multiple-access (TDMA) systems). The optimum assignment strategy must take account of the actual load spatial distribution, following the principle that a heavily loaded base station should hand off users to its neighboring stations in order to balance the load among cells [1].

The optimum assignment for the uplink of a CDMA system was first analyzed in [2] and [3]. In both papers, an iterative procedure is given that converges to the optimum assignment and its associated vector of transmitted powers, provided there is at least one feasible assignment to serve all users with the desired quality. In the case that no such assignment exists, the algorithm diverges. The "constrained power control" in [4] incorporates power constraints to assure convergence. The contribution of this paper is the following.

1) The results in [2] and [3] are extended to the more general setting, in which each user has a different signal-to-interference (SIR) target value in each base station.

2) A noniterative solution to the problem is given by means of an algorithm that finds the optimum assignment in a finite number of steps. This algorithm is efficient in the

Paper approved by G. E. Corazza, the Editor for Spread Spectrum of the IEEE Communications Society. Manuscript received February 24, 2001; revised May 22, 2002 and October 1, 2003. This work was supported in part by the Spanish Ministry of Education and Culture under a doctoral scholarship.

The authors are with the Polytechnic University of Madrid, 28040 Madrid, Spain (e-mail: 1mendo@grc.ssr.upm.es).

Digital Object Identifier 10.1109/TCOMM.2004.823623 sense that its computational complexity is polynomial in the number of users and base stations. Furthermore, it detects when there is no assignment that satisfies the system SIR requirements, taking into account power constraints.

The rest of the paper is organized as follows. In Section II, the system model is presented. In Section III, an analysis is made for a network with fixed assignment, and in Section IV, the results in [2] concerning the existence and characterization of the optimum assignment are extended to our more general setting. On the basis of these results, the algorithm for determination of the optimum assignment is derived in Section V, and its complexity is analyzed in Section VI. The capacity improvement effected by the algorithm is evaluated in Section VII, and applications are discussed in Section VIII. Conclusions are drawn in Section IX.

\section{SySTEM MODEL}

The uplink of a cellular CDMA system with single-user detection and pseudorandom coding sequences is considered. This means that other users' signals can be approximately regarded as white Gaussian noise. The cellular network consists of $M$ base stations ${ }^{1}$ that cover a geographical area in which, at a given instant, there are $K$ active users arbitrarily located. The system state is characterized by the following.

1) A $K \times M$ attenuation matrix $\boldsymbol{\alpha}=[\alpha(i, m)]$, where $\alpha(i, m)$ is the average (with respect to multipath fading) attenuation from user $i$ to cell site $m, 0<\alpha(i, m)<1$. This value includes all propagation factors, such as distance from mobile to base station and shadowing produced by obstacles, as well as antenna gains and terminal losses.

2) A $K \times M$ target SIR matrix $\breve{\gamma}=[\breve{\gamma}(i, m)]$, in which $\breve{\gamma}(i, m)>0$ is the target SIR for user $i$ in base station $m$. The target SIR is $\breve{\gamma}(i, m)=\left(E_{\mathrm{B}} / N_{0}\right)_{i, m} R_{i} / W$, where $\left(E_{\mathrm{B}} / N_{0}\right)_{i, m}$ is the required $E_{\mathrm{B}} / N_{0}$ for user $i$ in base station $m, W$ is the system bandwidth, and $R_{i}$ is the bit rate of user $i$. As usual in system-level analyses, the target SIR is defined as an average value with respect to multipath fading, and thus, it incorporates the effect of multipath.

3) An $M$-dimensional noise vector (column matrix) $\boldsymbol{\nu}=$ $[\nu(m)]^{T}$, where $\nu(m)>0$ is the background noise power (within the system bandwidth) at the $m$ th base station.

\footnotetext{
${ }^{1}$ For sectorized networks, each of the transceivers that serve a sector should
} be considered as a different base station. 
4) A $K$-dimensional assignment vector $\phi=[\phi(i)]^{T}$, where $\phi(i) \in\{1, \ldots, M\}$ denotes the base station to which the $i$ th user is assigned.

In a real system, the attenuations $\boldsymbol{\alpha}$ and SIR requirements $\breve{\gamma}$ vary with time, as a consequence of user mobility. Also, the number of active users in the system varies according to source burstiness (voice activity or data generation) and traffic processes (arrival and departure of calls or sessions). Throughout the paper, we assume that these parameters are fixed, thus modeling a "snapshot" of the system.

This model is identical to that of [2], except that we consider the possibility of different target SIR values for a mobile in each base station. This generalization is due to the fact that multipath characteristics and Doppler spread may be different from a given user to each of the base stations, resulting in different target SIRs. ${ }^{2}$

\section{System Description With FiXed AssignMENT}

Consider a system with $M$ base stations and $K$ users, characterized by matrices $\boldsymbol{\alpha}, \breve{\gamma}$, and $\boldsymbol{\nu}$, in an assignment $\phi$. Following [5] (with slight notational changes), we define $\mathbf{G}$ and $\boldsymbol{\gamma}$ as

$$
\begin{aligned}
G(m, n) & =\delta_{m, n}-\sum_{i \in \phi^{-1}(n)} \frac{\alpha(i, m)}{\alpha(i, n)} \gamma(i, n) \\
\gamma(i, m) & =\frac{\breve{\gamma}(i, m)}{1+\breve{\gamma}(i, m)} .
\end{aligned}
$$

Assuming $\operatorname{det}(\mathbf{G}) \neq 0$, we let $\mathbf{R}=\mathbf{H} \boldsymbol{\nu}$ with $\mathbf{H}=\mathbf{G}^{-1}$, and compute $\mathbf{P}=[P(i)]^{T}$ as

$$
P(i)=\frac{\gamma(i, \phi(i))}{\alpha(i, \phi(i))} R(\phi(i)), \quad i=1, \ldots, K .
$$

According to [5], $\mathbf{P}$ is the required transmitted power vector in assignment $\boldsymbol{\phi} ; \mathbf{R}=[R(m)]^{T}$ is the corresponding aggregate power vector, where

$$
R(m)=\sum_{i=1}^{K} \alpha(i, m) P(i)+\nu(m)
$$

represents the total received power at base station $m$, including background noise; and $\gamma(i, m)$ can be interpreted as a signal-tosignal-plus-interference ratio (SSIR). $\mathbf{G}$ will be referred to as the system matrix.

Under the assumption that $\mathbf{G}$ is nonsingular, there is a unique solution to the power control problem. However, this solution only has a physical meaning if all the power values $P(i)$ are nonnegative (maximum power limitations are considered in Section V-D). An assignment $\boldsymbol{\phi}$ is feasible (given $\boldsymbol{\alpha}, \boldsymbol{\gamma}$, and $\boldsymbol{\nu}$ ) [2] if all the components of the transmitted power vector $\mathbf{P}$, or equivalently, all the components of the aggregate power vector $\mathbf{R}$, are nonnegative. A feasible assignment is thus one in which all users can meet their requirements, provided that no transmit power limitations exist.

For a given assignment $\phi$, we define the foreign SSIR matrix $\Gamma=[\Gamma(i, m)]$ as follows: $\Gamma(i, m)$ is the SSIR at which the mo-

\footnotetext{
${ }^{2}$ Note that the practical limitation in the set of base stations $D(i)$ to which a mobile $i$ can be assigned, considered in [2], is included in our model by taking $\breve{\gamma}(i, m)=\infty$ for $m \notin D(i)$
}

bile $i$ would be received at cell site $m$ with transmitted powers equal to those required in assignment $\phi$

$$
\begin{aligned}
\Gamma(i, m) & =\frac{\alpha(i, m) P(i)}{\sum_{j=1}^{K} \alpha(j, m) P(j)+\nu(m)} \\
& =\frac{\gamma(i, \phi(i)) \alpha(i, m)}{\alpha(i, \phi(i))} \Lambda(\phi(i), m)
\end{aligned}
$$

where $\boldsymbol{\Lambda}=[\Lambda(l, m)]$ is a $M \times M$ matrix defined as $\Lambda(l, m)=$ $R(l) / R(m)$. Obviously, $\Gamma(i, \phi(i))=\gamma(i, \phi(i))$.

From the above definitions, it stems that $\mathbf{R}$ and $\mathbf{P}$ are functions of $\boldsymbol{\alpha}, \boldsymbol{\gamma}, \boldsymbol{\nu}$, and $\boldsymbol{\phi}$. The following proposition characterizes the feasible region of target SSIRs, and establishes that for any user $j \in\{1, \ldots, K\}$, an increment in $\gamma(j, \phi(j))$ within this region raises all aggregate powers $R(m)$ and all required powers $P(i)$. See the Appendix for proofs of propositions.

Proposition 1: For a system with $\boldsymbol{\alpha}, \boldsymbol{\nu}$, and $\boldsymbol{\phi}$ given, the feasible values of $\gamma(1, \phi(1)), \ldots, \gamma(K, \phi(K))$ lie in the finite region of $\mathbb{R}^{K}$, limited by the hypersurface $\operatorname{det}(\mathbf{G})=0$ and the coordinate hyperplanes $\gamma(1, \phi(1))=0, \ldots, \gamma(K, \phi(K))=0$. Within this region, $\mathbf{R}, \mathbf{P}, \boldsymbol{\Lambda}$, and $\boldsymbol{\Gamma}$ are $C^{1}$ functions of $\gamma(1, \phi(1)), \ldots, \gamma(K, \phi(K))$, and the partial derivatives of $R(m)$ and $P(i)$ with respect to these variables are positive, $\forall m \in\{1, \ldots, M\}, i \in\{1, \ldots, K\}$.

\section{OPTIMUM BASE-STATION ASSIGNMENT}

For a cellular network characterized by $M, K, \boldsymbol{\alpha}, \breve{\gamma}$, and $\nu$, and for a given optimality criterion, the problem arises to find the optimum base-station assignment $\phi$. The following fundamental result (given in [2] and [3] for $\gamma(i, m)$ independent of $m$ ) implies that all practical optimality criteria defined in terms of transmitted powers yield the same optimum assignment, and characterizes this assignment.

Proposition 2: For a system with $M$ base stations and $K$ users characterized by $\boldsymbol{\alpha}, \gamma, \boldsymbol{\nu}$ with at least one feasible assignment, there is an assignment $\phi^{\star}$ that simultaneously minimizes all the transmitted powers among the set of all feasible assignments. Furthermore, an assignment $\boldsymbol{\phi}^{\star}$, with matrices $\boldsymbol{\Gamma}^{\star}$ and $\boldsymbol{\Lambda}^{\star}$, is optimum (in this sense) if and only if any of the following equivalent conditions holds:

$$
\Gamma^{\star}(i, m) \leq \gamma(i, m) \quad \forall m \in\{1, \ldots, M\}, \quad \forall i \in\{1, \ldots, K\}
$$

$$
\begin{gathered}
\Lambda^{\star}(m, n) \leq \min _{i \in\left(\phi^{\star}\right)^{-1}(m)}\left\{\frac{\gamma(i, n) \alpha(i, m)}{\gamma(i, m) \alpha(i, n)}\right\} \\
\forall m, n \in\{1, \ldots, M\} .
\end{gathered}
$$

Note that, for any $i,(6)$ and (7) hold with equality for $m=$ $\phi^{\star}(i)$, at least.

\section{Algorithm FOR DETERMINATION OF OPTIMUM ASSIGNMENT AND TRANSMITTED POWERS}

We focus on the problem of determining the optimum assignment $\phi^{\star}$ (in the sense of Proposition 2), and its associated power vector $\mathbf{P}^{\star}$, for a system with $M, K, \boldsymbol{\alpha}, \breve{\gamma}$, and $\boldsymbol{\nu}$ given. This combinatorial optimization problem could, obviously, be solved by exhaustive search, with the computational burden growing ex- 
ponentially with $K$. Nevertheless, the structure of the problem can be exploited to substantially reduce the computational load. A finite algorithm that finds the solution with $O\left(M^{3} K^{2}\right)$ complexity is derived in this section.

The algorithm evolves appending users to the network one by one. In Section V-A, we outline a procedure to introduce a new user into the cellular network, which is formalized as an entry algorithm in Section V-B. In Section V-C the overall algorithm is defined as a succession of individual entries, and power constraints are considered in Section V-D.

\section{A. Entry of a New User}

Consider a system with $M$ base stations and $k$ users with attenuation matrix $\boldsymbol{\alpha}$, target SSIR matrix $\boldsymbol{\gamma}$, and noise vector $\boldsymbol{\nu}$ in a feasible assignment $\boldsymbol{\phi}$ with associated system matrix $\mathbf{G}$. Assume that a new user (not assigned yet to any base station) $k+1$ starts transmitting with power $p$. We will refer to the $k$ users in the system as internal users, and to the new user as the entering user. The latter is characterized by an attenuation vector $\boldsymbol{\alpha}_{k+1}=[\alpha(k+1,1) \cdots \alpha(k+1, M)]^{T}$ and a target SSIR vector $\gamma_{k+1}=[\gamma(k+1,1) \cdots \gamma(k+1, M)]^{T}$.

The signal transmitted by the entering user is seen at the $m$ th base station as additional noise with power $\alpha(k+1, m) p$. In order to meet the target SSIR for the internal users, the aggregate powers $^{3} \mathbf{R}(p)$ must be given as

$$
R(m ; p)=r(m)+s(m) p, \quad m=1, \ldots, M
$$

with $\mathbf{r}=\mathbf{H} \boldsymbol{\nu}, \mathbf{s}=\mathbf{H} \boldsymbol{\alpha}_{k+1}$. Note that $r(m)$ and $s(m)$ are positive, due to the feasibility of $\phi$.

The matrices $\boldsymbol{\Lambda}(p)=[\Lambda(l, m ; p)]$ and $\boldsymbol{\Gamma}(p)=[\Gamma(i, m ; p)]$ associated with $\phi$ are calculated from (5) as

$$
\begin{aligned}
& \Lambda(l, m ; p)=\frac{R(l ; p)}{R(m ; p)}=\frac{r(l)+s(l) p}{r(m)+s(m) p} \\
& \Gamma(i, m ; p)=\frac{\gamma(i, \phi(i)) \alpha(i, m)}{\alpha(i, \phi(i))} \Lambda(\phi(i), m ; p)
\end{aligned}
$$

$i=1, \ldots, k ; l, m=1, \ldots, M$. The SSIR that the entering user would experience if assigned to base station $m$ is

$$
\Gamma(k+1, m ; p)=\frac{\alpha(k+1, m) p}{r(m)+s(m) p}, \quad m=1, \ldots, M .
$$

Proposition 3: For a system with $M$ base stations and $k$ users in a given assignment $\phi, \Lambda(l, m ; p), \Gamma(i, m ; p)$ and $\Gamma(k+1, m ; p)$ are $C^{1}$ monotone functions of $p$, for all $l, m \in\{1, \ldots, M\}, i \in\{1, \ldots, K\}$ and $p \geq 0$.

For a given assignment $\phi$ and a given user $b \in\{1, \ldots, k\}$, its required power $P(b ; p)$ has an incrementally linear variation with $p$, according to (8) and (3). We define the power diagram for user $b$ to be a graphical representation of $P(b ; p)$ as a function of $p$, with the assignment $\phi$ as a parameter (see Fig. 1). Each assignment generates a line in this power diagram. Of course, we are only interested in feasible assignments, whose slope and $P(b ; p)$-intercept are positive. For a given value of

${ }^{3}$ In the following, we explicitly show the dependence on $p$ where convenient.

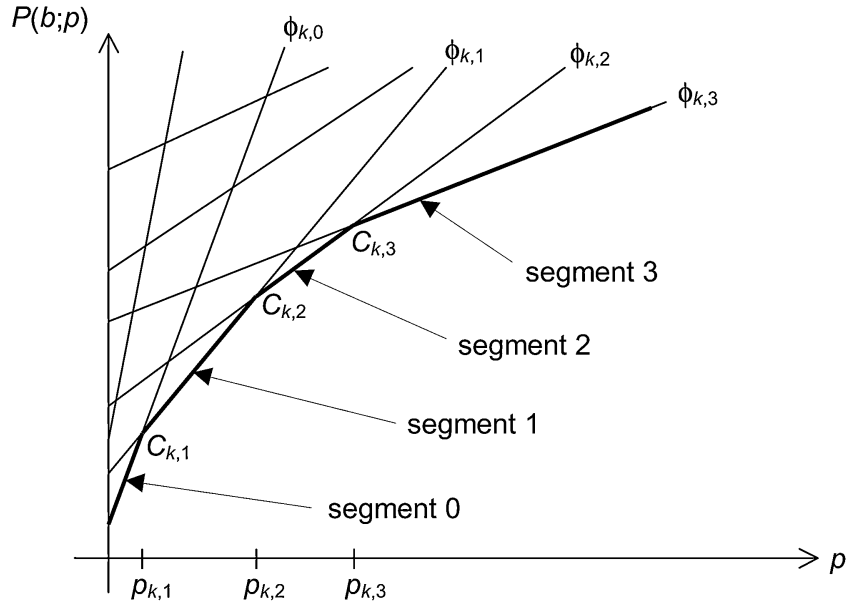

Fig. 1. Entry process and minimum-power line (example with $\bar{T}_{k}=3$ ).

$p$, the optimum assignment for the $k$ internal users is that corresponding to the line with minimum (positive) ordinate. According to Proposition 2, this assignment (line) must be the same in every user's power diagram. In addition, the following result holds.

Proposition 4: Consider a system with $k$ internal users and an entering user. Let $\boldsymbol{\phi}^{\star}$ be the optimum assignment for $p=p_{0}$, and $\boldsymbol{\Gamma}^{\star}(p)$ its associated foreign SSIR matrix as a function of $p$. As $p$ is increased from $p_{0}$, the first intersection point $C$ of $\boldsymbol{\phi}^{\star}$ occurs with the same assignment $\phi^{+}$in the power diagram of every user $b$. At this intersection point, with abscissa $p=p_{C}$

$$
\Gamma^{\star}\left(h, \phi^{+}(h) ; p_{C}\right)=\gamma\left(h, \phi^{+}(h)\right) \quad \forall h \in\{1, \ldots, k\} .
$$

The procedure for a new user's entry is based on Proposition 4 . We begin with the $k$ internal users in an optimum assignment $\phi_{k, 0}$. Suppose that the entering user continuously increases its transmitted power $p$ beginning from zero, and during that process, the internal users' transmitted powers are adjusted according to (8) and (3). Observe that this gradually raises all transmitted powers. If, as $p$ increases, $\Gamma_{k, 0}(i, m ; p)$ reaches $\gamma(i, m)$ for any user $i$ in any cell $m \neq \phi_{k, 0}(i)$ (note that $\left.\Gamma_{k, 0}(i, m ; 0) \leq \gamma(i, m)\right)$, user $i$ is switched to cell $m$. While $p$ continues increasing, if the new user's SSIR at some cell $m$ equals its target value $\gamma(k+1, m)$, the process finishes, with the new user assigned to that cell. In the following, we show that the resulting assignment is optimum for the $k+1$ users, and investigate the behavior when no feasible assignment exists for the $k+1$ users.

The process begins in assignment $\phi_{k, 0}$, which is optimum for $p=0$, and has an associated system matrix $\mathbf{G}_{k, 0}, \mathbf{r}_{k, 0}=\mathbf{H}_{k, 0} \boldsymbol{\nu}$ and $\mathbf{s}_{k, 0}=\mathbf{H}_{k, 0} \boldsymbol{\alpha}_{k+1}$. As $p$ increases, $\boldsymbol{\phi}_{k, 0}$ remains as the optimum assignment until the first intersection point $C_{k, 1}$ is reached, at abscissa $p=p_{k, 1}$, as shown in Fig. 1 . At $C_{k, 1}$, the foreign SSIRs for one or more users in some respective cells other than their assigned ones equal the corresponding target values (Proposition 4), and the referred users are switched to those base stations, yielding the new assignment vector $\boldsymbol{\phi}_{k, 1}$. We now proceed along the $\boldsymbol{\phi}_{k, 1}$ line in the power diagram, with associated vectors $\mathbf{r}_{k, 1}, \mathbf{s}_{k, 1}$. This new assignment is optimum for $p_{k, 1} \leq p \leq p_{k, 2}$. At $C_{k, 2}$ there is a new assignment change 


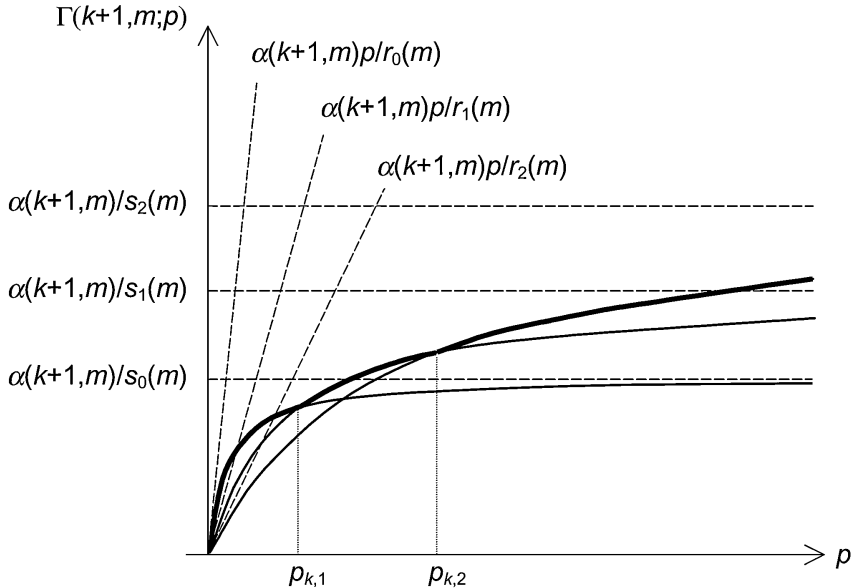

Fig. 2. Evolution of new user's SSIR in a given cell.

to $\phi_{k, 2}$, and the process continues. This procedure follows the optimum assignment for the internal users as $p$ increases, given by the minimum-power line drawn thick in Fig. 1. It can be seen that this is a polygonal (i.e., piecewise incrementally linear), monotone increasing, convex $(\cap)$ line. Since every point $C_{k, 1}, C_{k, 2}, \ldots$ occurs at the same abscissa in the power diagram of every user (Proposition 4) and the assignment changes take place precisely at these points, we may call them transition points. The $t$ th segment of the minimum-power line is delimited by $C_{k, t}$ and $C_{k, t+1}$, and corresponds to assignment $\phi_{k, t}$.

The variation of the new user's SSIR in a cell $m$ during this entry process, $\Gamma(k+1, m ; p)$, is now analyzed. For a given assignment, this variation is expressed by (11). The number of feasible assignments is upper bounded by $M^{k}$, and, in general, not all of them take part in the minimum-power line, as depicted in Fig. 1. Therefore, as $p$ is increased toward infinity, there are a finite number of transitions $\bar{T}_{k}<M^{k}{ }^{4}$ Assuming that the new user eventually enters, this happens after a number $T_{k}$ of transitions, with $T_{k} \leq \bar{T}_{k}$. For any $t \in\left\{1, \ldots, \bar{T}_{k}\right\}$, we have $r_{k, t+1}(m)>r_{k, t}(m)$ and $s_{k, t+1}(m)<s_{k, t}(m)$, for otherwise, the intersection point $C_{k, t}$ would not lie in the $p>0$ region. Therefore, the variation of $\Gamma(k+1, m ; p)$ along the different segments has the general shape depicted in Fig. 2.

From the foregoing discussion, it is clear that $\Gamma(k+1, m ; p)$ is monotone increasing with $p$, and

$$
\lim _{p \rightarrow \infty} \Gamma(k+1, m ; p)=\alpha(k+1, m) / s_{k, \bar{T}_{k}}(m) .
$$

Hence, the new user is able to enter if and only if

$$
\min _{m \in\{1, \ldots, M\}}\left\{s_{k, \bar{T}_{k}}(m) \frac{\gamma(k+1, m)}{\alpha(k+1, m)}\right\}<1 .
$$

If (14) is satisfied, the new user is eventually accepted in some cell. This power raising and assignment-switching process tracks the optimum assignment for the internal users as $p$ varies, and stops as soon as the new user reaches its target in some cell. These two features, together with the monotonic increasing

\footnotetext{
${ }^{4} \mathrm{As}$ will be seen in Section VI-A, this upper bound is very loose; however, it suffices at this point to show that $\bar{T}_{k}$ is finite.
}

character of the minimum-power line for any internal user, assure that the power for all internal users is minimum at the end of the process. The power for the new user is also the minimum possible. This is because we stop increasing $p$ as soon as possible, while following the optimum assignment for the internal users, and if we selected any other assignment, the powers transmitted by the internal users would be larger, and thus, $\Gamma(k+$ $1, m ; p)$ would be lower in every cell $m$. Hence, the optimum assignment for the $k+1$ users results at the end of the process. On the other hand, if (14) is not satisfied, there is no feasible assignment for the $k+1$ users.

\section{B. Algorithm for the Entry of a New User}

We now develop an entry algorithm that replaces the continuous variation of $p$ by discrete changes. We begin with some results that will be useful in the development of the entry algorithm and in the computational complexity analysis of Section VI.

Suppose that we are in the optimum assignment $\phi_{k, t}$ at segment $t$ of the entry process described in Section $\mathrm{V}$-A with $k$ internal users. At $C_{k, t+1}, L \geq 1$ users, identified as $i_{1}, \ldots, i_{L}$, are switched to foreign base stations, where they have reached their respective target SSIRs. If, at this transition, user $i_{l}, l \in$ $\{1, \ldots, L\}$ is switched to $m_{l} \neq \phi_{k, t}\left(i_{l}\right), \Gamma_{k, t}\left(i_{l}, m_{l} ; p\right)$ must be monotone nondecreasing with $p$ (because $\Gamma_{k, t}\left(i_{l}, m_{l} ; p_{k, t}\right) \leq$ $\gamma\left(i_{l}, m_{l}\right), \Gamma_{k, t}\left(i_{l}, m_{l} ; p_{k, t+1}\right)=\gamma\left(i_{l}, m_{l}\right)$, and $\Gamma_{k, t}\left(i_{l}, m_{l} ; p\right)$ is a monotone function of $p$ ). Equation (10) then implies that $\Lambda_{k, t}\left(\phi_{k, t}\left(i_{l}\right), m_{l} ; p\right)$ is also monotone nondecreasing, and so is $\Gamma_{k, t}\left(i, m_{l} ; p\right)$ for all users $i \in \phi_{k, t}^{-1}\left(i_{l}\right)$. This proves that the increasing character of the foreign SSIR depends only on the "home" and "foreign" base stations, and not on the particular user; and that if a user assigned to a cell $m$ has a foreign SSIR in cell $n$ that increases with $p$, users from cell $n$ have decreasing SSIRs in cell $m$. Therefore, users can only change from $m$ to $n$ if $\Lambda(m, n ; p)$ increases with $p$. If $\Lambda(m, n ; p)$ is equal to a constant $c$, users from cell $m$ cannot change to $n$ and vice versa, unless $c \gamma(i, m) \alpha(i, n)=\gamma(i, n) \alpha(i, m)$ for some $i$. In this case, user $i$ can be assigned to either of the two cells without affecting the rest of the system, and this can be disregarded as a real change in the system. Thus, for a given pair of cells $m \neq n$ in segment $t$, users only change either from $m$ to $n$ or from $n$ to $m$, depending on the sign of $\partial \Lambda_{k, t}(m, n ; p) / \partial p$. The following proposition states that this unidirectional feature is maintained for different segments of the entry process, i.e., if a user is switched from cell $m$ to $n$ at transition $t$, there may be no subsequent transitions from cell $n$ to $m$.

Proposition 5: For all $t, u \in\left\{1, \ldots, \bar{T}_{k}\right\}$

$$
\operatorname{sgn} \frac{\partial \Lambda_{k, t}(m, n ; p)}{\partial p}=\operatorname{sgn} \frac{\partial \Lambda_{k, u}(m, n ; p)}{\partial p} .
$$

This result allows us to define the relation (or simple directed graph)

$$
\begin{array}{r}
\mathrm{L}_{k}=\left\{(m, n) \in\{1, \ldots, M\}^{2} \mid \partial \Lambda_{k, t}(m, n ; p) / \partial p \geq 0\right. \\
\text { for any } \left.t=0, \ldots, \bar{T}_{k}\right\}
\end{array}
$$

with the following properties. 
Proposition 6: $\mathrm{L}_{k}$ is a total order relation, i.e., it is antisymmetric, transitive, and $\forall m, n \in\{1, \ldots, M\}$ either $(m, n) \in \mathrm{L}_{k}$ or $(n, m) \in \mathrm{L}_{k}$.

The foregoing results allow a formulation of the entry algorithm for a new user in a $k$-user system. Suppose that every pair of cells $(m, n)$ is initially classified according to whether $\Lambda_{k, t}(m, n ; p)$ increases (cell $m$ tends to transfer users to $n$ ) or decreases with $p$ (vice versa). Since this is independent of $t$, classification can be made at $t=0$, for instance. Pairs for which $\Lambda_{k, t}(m, n ; p)$ is constant can be simply eliminated from successive development. Let the system be in the optimum assignment $\phi_{k, t}$ at the initial point $p=p_{k, t}$ of segment $t$ of the entry process. For every $(m, n)$ with $\partial \Lambda_{k, t}(m, n ; p) / \partial p>0$, we define

$$
i_{k, t}^{m, n}=\min _{i \in \phi_{k, t}^{-1}(m)}\left\{\frac{\gamma(i, n) \alpha(i, m)}{\gamma(i, m) \alpha(i, n)}\right\} .
$$

This is the "candidate" user for transition from $m$ to $n$, i.e., $i_{k, t}^{m, n}$ will be the first user that changes from $m$ to $n$, if further transitions take place between this pair of cells. A set with a maximum of $M(M-1) / 2$ candidates $\left\{i_{k, t}^{m, n}\right\}$ is thus obtained, and for each, we calculate the value $p_{k, t}^{m, n}$ at which the transition would take place. This value is obtained from the condition $\Gamma_{k, t}\left(i_{k, t}^{m, n}, n ; p_{k, t}^{m, n}\right)=\gamma\left(i_{k, t}^{m, n}, n\right)$, and is given by (18), shown at the bottom of the page. If $p_{k, t}^{m, n}$ is positive, then it is necessarily greater than $p_{k, t}$, because $\Gamma_{k, t}\left(i_{k, t}^{m, n}, n ; p_{k, t}\right)<$ $\gamma\left(i_{k, t}^{m, n}, n\right)$ and $\Gamma_{k, t}\left(i_{k, t}^{m, n}, n ; p\right)$ is a continuous, increasing function of $p$ for $p \geq 0$. A negative or infinite $p_{k, t}^{m, n}$ means that

$$
\begin{aligned}
\lim _{p \rightarrow \infty} \Gamma_{k, t}\left(i_{k, t}^{m, n}, n ; p\right) & =\frac{s_{k, t}(m) \gamma\left(i_{k, t}^{m, n}, m\right) \alpha\left(i_{k, t}^{m, n}, n\right)}{s_{k, t}(n) \alpha\left(i_{k, t}^{m, n}, m\right)} \\
& \leq \gamma\left(i_{k, t}^{m, n}, n\right)
\end{aligned}
$$

and hence, in assignment $\boldsymbol{\phi}_{k, t}$, the candidate user $i_{k, t}^{m, n}$ can never be switched to cell $n$, regardless of the value of $p$. (Moreover, it can be shown that a negative $p_{k, t}^{m, n}$ is necessarily lower than $-r_{k, t}(n) / s_{k, t}(n)$.) The next transition in the power diagram takes place at abscissa

$$
p_{k, t+1}=\min _{m, n}\left\{p_{k, t}^{m, n} \mid p_{k, t}^{m, n}>0, m, n=1, \ldots, M\right\}
$$

and corresponds to a (single) transition of user $i_{k, t+1}$ from cell $m_{k, t+1}$ to $n_{k, t+1}$, where $m_{k, t+1}$ and $n_{k, t+1}$ are the arguments that minimize the above expression, and $i_{k, t+1} \triangleq i_{k, t}^{m_{k, t+1}, n_{k, t+1}}$. A multiple transition will take place if the minimum is reached by several pairs of cells, or if it is reached by several users for the same pair of cells. Observe that a multiple transition can simply be treated in our framework as several coincident single transitions. If there are no values $p_{k, t}^{m, n}>0$, no further transitions can take place (i.e., we are already in segment $t=\bar{T}_{k}$ ) and $p_{k, t+1}$ is undefined.

Likewise, for every $m$, we calculate the value $\hat{p}_{k, t}^{m}$ at which the entering user would achieve its target SSIR in assignment $\phi_{k, t}$. This value is determined by the condition $\Gamma_{k, t}\left(k+1, m ; \hat{p}_{k, t}^{m}\right)=\gamma(k+1, m)$, and equals

$$
\hat{p}_{k, t}^{m}=\frac{\gamma(k+1, m) r_{k, t}(m)}{\alpha(k+1, m)-\gamma(k+1, m) s_{k, t}(m)} .
$$

A positive $\hat{p}_{k, t}^{m}$ is necessarily greater than $p_{k, t}$. If

$$
\lim _{p \rightarrow \infty} \Gamma_{k, t}(k+1, m ; p)=\frac{\alpha(k+1, m)}{s_{k, t}(m)} \leq \gamma(k+1, m)
$$

then the entering user cannot be accepted in cell $m$ with the current assignment vector, and the obtained $\hat{p}_{k, t}^{m}$ is negative or infinite. We define

$$
\hat{p}_{k, t}=\min _{m}\left\{\hat{p}_{k, t}^{m} \mid \hat{p}_{k, t}^{m}>0, \quad m=1, \ldots, M\right\}
$$

assuming that the set in the right-hand side is nonempty. The minimum is reached for an argument $\hat{m}_{k, t}$, which represents the (first) cell that accepts the entering user in the current segment. If several cells minimize the above expression, all of them are equally capable of serving the entering user.

Once $p_{k, t+1}$ and $\hat{p}_{k, t}$ have been calculated, the following situations can be found.

i) $\quad p_{k, t+1}>\hat{p}_{k, t}>0$ : the entering user is assigned to cell $\hat{m}_{k, t}$ before the next transition takes place (i.e., $t=T_{k}<\bar{T}_{k}$ ), and the entry algorithm finishes successfully.

i') $\quad \hat{p}_{k, t}>0$ and $p_{k, t+1}$ is infinite or undefined: no more transitions are possible, but the entering user is admitted in cell $\hat{m}_{k, t}$ in the current segment $\left(t=T_{k}=\right.$ $\bar{T}_{k}$ ), and the algorithm finishes successfully.

ii) $\quad \hat{p}_{k, t}>p_{k, t+1}>0$ : transition $t+1$ takes place before the new user enters.

ii') $\quad p_{k, t+1}>0$ and $\hat{p}_{k, t}$ is infinite or undefined: the new user could not be accepted in the current segment, but a $(t+1)$ th transition takes place (the new user will perhaps be accepted later).

iii) $\hat{p}_{k, t}$ and $p_{k, t+1}$ are both either infinite or undefined: neither the new user is able to enter in the current segment nor can a transition take place $\left(t=\bar{T}_{k}\right)$. Hence, there is no feasible assignment for the $k+1$ users, and the algorithm terminates unsuccessfully.

The entry algorithm with $k$ internal users is thus expressed as follows:

1) initialize $t=0$;

2) compute $p_{k, t+1}$ and $\hat{p}_{k, t}$;

$$
p_{k, t}^{m, n}=\frac{\gamma\left(i_{k, t}^{m, n}, n\right) \alpha\left(i_{k, t}^{m, n}, m\right) r_{k, t}(n)-\gamma\left(i_{k, t}^{m, n}, m\right) \alpha\left(i_{k, t}^{m, n}, n\right) r_{k, t}(m)}{\gamma\left(i_{k, t}^{m, n}, m\right) \alpha\left(i_{k, t}^{m, n}, n\right) s_{k, t}(m)-\gamma\left(i_{k, t}^{m, n}, n\right) \alpha\left(i_{k, t}^{m, n}, m\right) s_{k, t}(n)}
$$


3) decide, depending on these values, whether to accept the entering user and terminate successfully [situations (i) and (i')], increment $t$ and go back to 2 [situations (ii) and (ii')], or terminate unsuccesfully [situation (iii)].

\section{Algorithm for Optimum Assignment}

The optimum assignment for a given set of $K$ users with parameters $\boldsymbol{\alpha}, \gamma$, and $\boldsymbol{\nu}$ can be determined by applying the entry algorithm of Section V-B $K$ times, one for each of the users, selected in an arbitrary order. The algorithm thus consists of $K$ entry steps. At the $k$ th entry step, the $(k+1)$ th user is appended to a $k$-user system. The process finishes successfully when the $K$ th user has entered, or stops prematurely if situation (iii) is encountered at any entry step. Observe that (iii) at the $k$ th entry means that no feasible assignment exists for users $1, \ldots, k$, neither does it for users $1, \ldots, K$.

\section{Power Constraints}

The proposed algorithm has the property that the transmitted powers increase as the algorithm progresses (i.e., as transitions and entries take place). As a consequence, it lends itself naturally to transmitted power constraints: the algorithm simply stops whenever a required power exceeds the maximum allowable power of that user, meaning that no feasible assignment exists that is compatible with the power constraints.

\section{COMPLEXITY ANALYSIS}

The computational cost of the proposed algorithm is now evaluated, in terms of the number of arithmetic additions/subtractions $(\mathrm{a} / \mathrm{s})$, multiplications/divisions $(\mathrm{m} / \mathrm{d})$, and pairwise comparisons (operations that involve no arithmetic are not taken into account).

In this analysis, it is assumed that there is at least one feasible assignment compatible with the power constraints for the $K$ users, so that the algorithm succeeds in finding an optimum assignment. A worst-case analysis is carried out in Section VI-A to obtain an upper bound in the number of operations. Simulation is then used in Section VI-B to assess average complexity.

Since we are not interested in the exact number of computations, but rather in its order of magnitude for $M$ and $K$ large, we will make use of multivariate asymptotic notation [6, Sec. 3.5] $f(u, v)=O(g(u, v)) \Leftrightarrow f(u, v) \leq c g(u, v)$ for some positive constant $c$ and sufficiently high values of both parameters $u$ and $v$.

\section{A. Complexity Upper Bound}

The number of transitions in the $(k+1)$ th entry, $T_{k}$, can be upper bounded as $T_{k} \leq \bar{T}_{k} \leq(M-1) k$. This is a consequence of Proposition 6: if a transition from $m$ to $n$ takes place, no user from $n$ can "go back" to $m$ during the current entry. The least favorable situation is one in which all internal users are initially assigned to a cell and visit all other cells in the same order; this yields the maximum number of transitions. Since multiple transitions are simply two or more single transitions that coincide, the bound holds when multiple transitions are accounted for according to their multiplicity order.

When the $(k+2)$ th entry begins, the new vectors $\mathbf{r}_{k+1,0}$ and $\mathbf{s}_{k+1,0}$ must be calculated. For $k=0$, we initialize $\mathbf{G}_{0,0}=$ $\mathbf{I}_{M}, \mathbf{r}_{0,0}=\boldsymbol{\nu}$, and $\mathbf{s}_{0,0}=\boldsymbol{\alpha}_{1}$. Since $\mathbf{G}_{k+1,0}$ differs from $\mathbf{G}_{k, T_{k}}$ only in column $\hat{m}_{k, t}$, it can be expressed as a rank-1 modification of this matrix

$$
\begin{aligned}
& \mathbf{G}_{k+1,0}=\mathbf{G}_{k, T_{k}}+[\alpha(k, 1) \quad \cdots \quad \alpha(k, M)]^{T}
\end{aligned}
$$

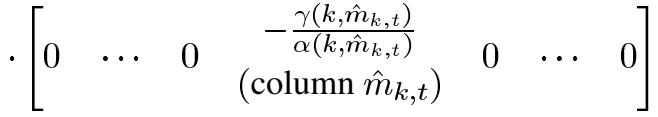

and therefore, the new vectors $\mathbf{r}_{k+1,0}$ and $\mathbf{s}_{k+1,0}$ can be computed in approximately $2\left(M^{2}+M+1 / 3\right)=O\left(M^{2}\right) \mathrm{m} / \mathrm{d}$ and a comparable number of a/s [7, Th. (3.63)(d)] (the fact that the row vector in (22) has only one nonzero element can be exploited to reduce the number of operations, but it remains $O\left(M^{2}\right)$ ).

Similarly, at the $(t+1)$ th single transition of the $(k+1)$ th entry, in which user $i_{k, t+1}$ is switched from cell $m_{k, t+1}$ to $n_{k, t+1}, \mathbf{G}_{k, t+1}$ can be obtained from $\mathbf{G}_{k, t}$, as given by (23) at the bottom of the page, with roughly the same computational effort as in the previous paragraph. For multiple transitions, the update (23) must be performed once for each involved user.

The determination of the candidate users in each segment of each entry can be done efficiently, if for every $(m, n)$ with $\partial \lambda(m, n ; p) / \partial p>0$, all $K$ users are initially sorted in increasing order of $(\gamma(i, n) \alpha(i, m)) /(\gamma(i, m) \alpha(i, n))$. Identifying which cell tends to transfer users to the other requires two multiplications and one comparison. Since cell $m$ tends to transfer users to cell $n$, we may call the former a "donor" cell and the latter an "acceptor" cell. We thus make $M(M-1) / 2$ ordered lists, one for each pair of cells. It is well known that $O(K \log K)$ comparisons are sufficient to order a set of $K$ elements [8, Th. 9.5]. Therefore, the cost of the initial sorting is $c_{0}=O\left(M^{2} K \log K\right)$ comparisons. Each list consists of $K$ positions, one for each user, although, in a given instant, not all users will be necessarily present in a particular cell. If, at a given entry and transition, a user moves from cell $m$ to $n$, it must be marked as present in all lists that involve $n$ as donor cell. Note that this involves no comparisons; the user simply occupies its precomputed position. The candidate user in each list is the first that is present, which can be determined without further arithmetic comparisons (by means of a linked list).

Each $p_{k, t}^{m, n}$ value requires a fixed number of operations, and so does each $\hat{p}_{k, t}^{m}$ value. Since there are $M(M-1) / 2$ of the

$$
\begin{aligned}
& \mathbf{G}_{k, t+1}=\mathbf{G}_{k, t}+\left[\alpha\left(i_{k, t+1}, 1\right) \quad \cdots \quad \alpha\left(i_{k, t+1}, M\right)\right]^{T}
\end{aligned}
$$

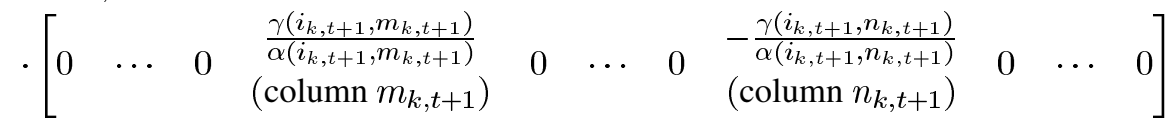


former and $M$ of the latter values per entry, and a maximum of $(M-1) k$ entries, the computational cost is $O\left(M^{3} k\right) \mathrm{m} / \mathrm{d}$ and a similar number of a/s.

The minimum element of a set can be determined with a number of comparisons equal to the number of elements minus one [8, Th. 9.2], and hence, the determination of $p_{k, t+1}$ from the values $p_{k, t}^{m, n}$ requires $M(M-1) / 2$ comparisons against zero plus, at most, $M(M-1) / 2$ comparisons for the minimum positive value, that is, $M(M-1)$ or fewer comparisons. Likewise, $\hat{p}_{k, t}$ can be found from the values $\hat{p}_{k, t}^{m}$ in, at most, $2 M$ comparisons.

Assuming that the algorithm concludes successfully, the transmitted powers $P(i), i=1, \ldots, K$ must be computed at the end. $P(K)$ is directly $\hat{p}_{K-1, T_{K-1}} ; \mathbf{R}$ is computed as $\mathbf{r}_{K-1, T_{K-1}}+\mathbf{s}_{K-1, T_{K-1}} \hat{p}_{K-1, T_{K-1}}$ with $M$ multiplications and $M$ additions, and finally, $P(i), i=1, \ldots, K-1$ are calculated from $R\left(\phi_{K-1, T_{K-1}}(i)\right)$ as in (3), with $2 K-2$ $\mathrm{m} / \mathrm{d}$. Hence, these final calculations require $a_{\mathrm{F}}=M$ a/s and $b_{\mathrm{F}}=M+2 K-2 \mathrm{~m} / \mathrm{d}$.

For the worst-case $(k+1)$ th entry involving $(M-1) k$ single transitions (with some of them perhaps grouped in multiple transitions), the following computations must be made.

1) $M(M-1) \mathrm{m} / \mathrm{d}$ and $M(M-1) / 2$ comparisons to identify donor and acceptor for all pairs of cells.

2) One update of vectors $\mathbf{r}, \mathbf{s}$ from the previous entry and one update at each transition, with a maximum of $(M-1) k$. This amounts to $O\left(M^{3} k\right)$ a/s and $O\left(M^{3} k\right) \mathrm{m} / \mathrm{d}$.

3) $(M-1) k$ computations of $M(M-1) / 2$ values $p_{k, t}^{m, n}$ and $M$ values $\hat{p}_{k, t}$, therefore, $O\left(M^{3} k\right)$ a/s and $O\left(M^{3} k\right) \mathrm{m} / \mathrm{d}$.

4) At most, $M(M-1)$ comparisons to obtain $p_{k, t+1}$, and at most, $2 M$ to obtain $\hat{p}_{k, t}$ in each segment. This yields $M\left(M^{2}-1\right)(k-1)=O\left(M^{3} k\right)$ comparisons.

The number of operations in the $(k+1)$ th entry is thus $a_{k}=$ $O\left(M^{3} k\right) \mathrm{a} / \mathrm{s}, b_{k}=O\left(M^{3} k\right) \mathrm{m} / \mathrm{d}$, and $c_{k}=O\left(M^{3} k\right)$ comparisons.

When the $(k+1)$ th entry process finishes (successfully), and $k<K-1$, we proceed with the $(k+2)$ th entry. Including the initial sorting and the final calculations, the total number of operations is $\sum_{k=0}^{K-1} a_{k}+a_{F}=O\left(M^{3} K^{2}\right)$ a/s, $\sum_{k=0}^{K-1} b_{k}+b_{F}=$ $O\left(M^{3} K^{2}\right) \mathrm{m} / \mathrm{d}$, and $c_{0}+\sum_{k=0}^{K-1} c_{k}=O\left(M^{3} K^{2}\right)$ comparisons.

A few remarks are convenient. Although the modified-matrix approach used to update the vectors $\mathbf{r}$ and $\mathbf{s}$ reduces the number of computations from $O\left(M^{3}\right)$ to $O\left(M^{2}\right)$, it may exhibit the problem of error accumulation, which advises to periodically "reset" the errors by solving for these vectors using (partialpivoting) Gauss elimination. This requires $O\left(M^{3}\right)$ arithmetic operations (a/s and $\mathrm{m} / \mathrm{d})$ and $O\left(M^{2}\right)$ arithmetic comparisons for the pivot selection [9, Alg. 3.4.1]. If this is done once per entry, the total associated computational load will be $O\left(M^{3} K\right)$ a/s and $\mathrm{m} / \mathrm{d}$, and $O\left(M^{2} K\right)$ comparisons. Therefore, this periodic error truncation is not computationally costly.

In a real setting, for a given $K$, path attenuations and target SSIR values vary in a rather unpredictable manner, and hence, are well modeled as random variables. In this case, under very mild conditions (boundedness of the joint probability density function of $\boldsymbol{\alpha}$ and $\boldsymbol{\gamma}$ is sufficient, as it is easily shown), the following "degenerate" events have probability $0: \operatorname{det}(\mathbf{G})=0(\mathbf{G}$

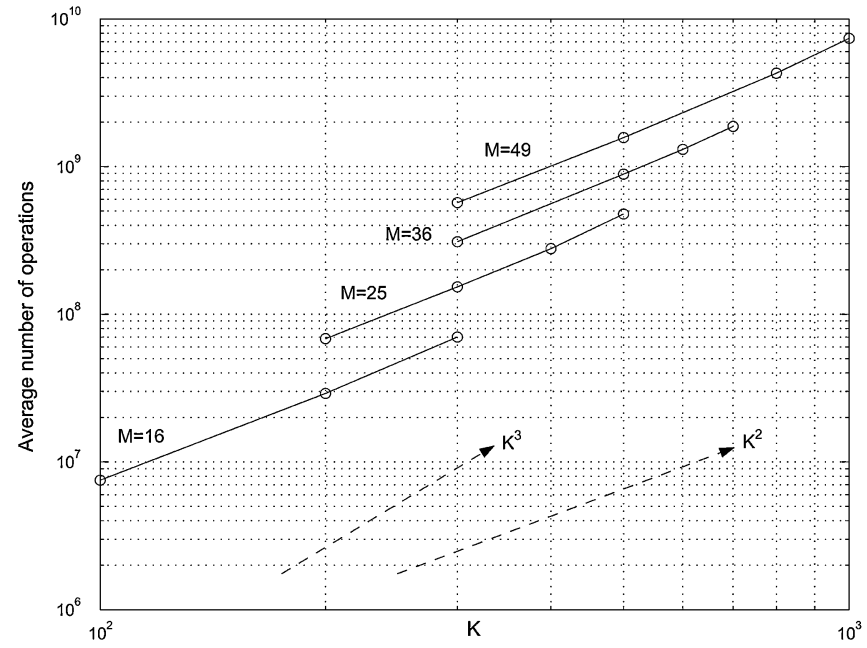

Fig. 3. Variation of the average number of operations with $K$.

singular); $\partial \lambda(m, n ; p) / \partial p=0$ (no need to consider exchanges between cells $m$ and $n$ ); $H(m, n)=0$ (users in cell $m$ unaffected by thermal noise in cell $n) ; \Gamma^{\star}(i, m)=\gamma(i, m), i \neq$ $\phi^{\star}(i)$, for $\boldsymbol{\phi}^{\star}$ optimum (optimum assignment not unique); and

$$
\frac{\gamma(i, n) \alpha(i, m)}{\gamma(i, m) \alpha(i, n)}=\frac{\gamma(j, n) \alpha(j, m)}{\gamma(j, m) \alpha(j, n)}, i, j \in \phi_{k, t}^{-1}(m)
$$

(possible multiple transition from $m$ to $n$ ).

\section{B. Average Complexity}

A simulation analysis has been carried out in order to assess the average complexity of the algorithm in a practical scenario. A square grid of $\sqrt{M} \times \sqrt{M}$ omnidirectional base stations is considered, with 1-km separation between cell sites. For a given $K$, users are randomly generated within the considered area, and the described algorithm (with direct Gauss elimination once per entry) is applied. Two classes of service are considered, with respective bit rates 12 and $64 \mathrm{~kb} / \mathrm{s}$. Target SIRs are generated as normal independent random variables with mean $-20.2 \mathrm{~dB}$ for the $12 \mathrm{-kb} / \mathrm{s}$ service and $-12.9 \mathrm{~dB}$ for the $64-\mathrm{kb} / \mathrm{s}$ service, corresponding to $E_{\mathrm{B}} / N_{0}=6 \mathrm{~dB}$ and $W=5 \mathrm{MHz}$ in both cases; and standard deviation $1.5 \mathrm{~dB}$. Half of the $K$ mobiles use each type of service. Path loss in decibels is calculated as $144.4+38.4 \log d$, where $d$ is the distance in kilometers. A normal shadowing component is included with standard deviation $8 \mathrm{~dB}$, assuming independent shadowing for each mobile-base link. The total effect of antenna gains and terminal losses is $9 \mathrm{~dB}$, and the noise figure is $4 \mathrm{~dB}$. Transmission power at the mobile is unlimited.

Simulations have been performed for different combinations of $M$ and $K$, with 20 successful (meaning that all users are accepted) snapshots in each case. It has been observed that $K / M \approx 20$ is a practical limit for the considered scenario. ${ }^{5}$ The total number of operations (aggregating the three categories) is represented in Figs. 3 and 4 . These values suggest that the average complexity is approximately $O\left(M^{2} K^{2}\right)$. The

${ }^{5}$ It can be checked that the pole capacity of a single cell, with the given mix of services and no SIR fluctuations, is 34 users. 


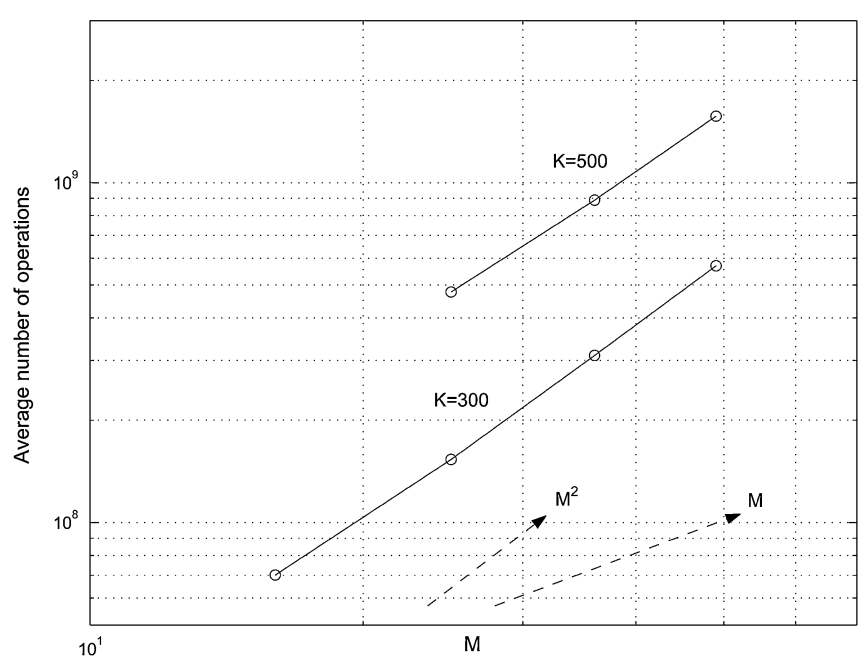

Fig. 4. Variation of the average number of operations with $M$.

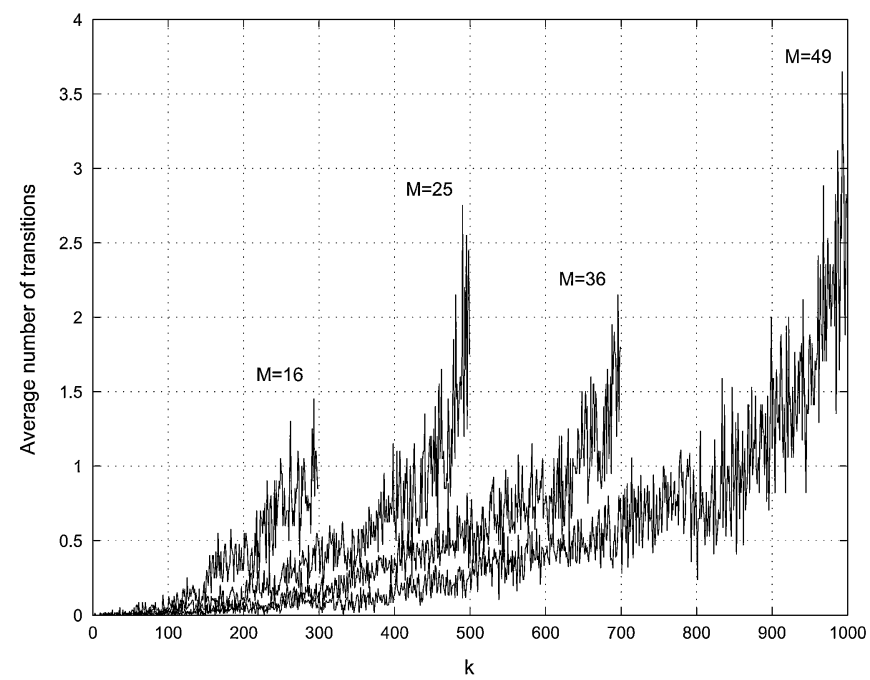

Fig. 5. Average number of transitions.

reduction in computational burden is explained from the results in Fig. 5, which show that the upper bound $T_{k} \leq(M-1) k$ is very pessimistic in practice.

\section{CAPACITY ANALYSIS}

The described algorithm, which minimizes transmitted powers, is also optimum with respect to capacity in the following sense. If the users are arbitrarily ordered and added one by one, the proposed algorithm yields the maximum number of accepted users (before power limitations or infeasibility are reached), compared against any other assignment criterion, for the same ordering of users. Observe that this also applies to the algorithm described in [2] and [3], in which the optimum assignment is also arrived at (by a different procedure).

The question arises as to how large the capacity improvement is. This topic was partially addressed in [2], illustrating the cellbreathing effect, but no attempt was made to obtain numerical results on system capacity. Also, regarding our algorithm, it is important to characterize the degradation caused by estimation errors in its input parameters, namely attenuations and target

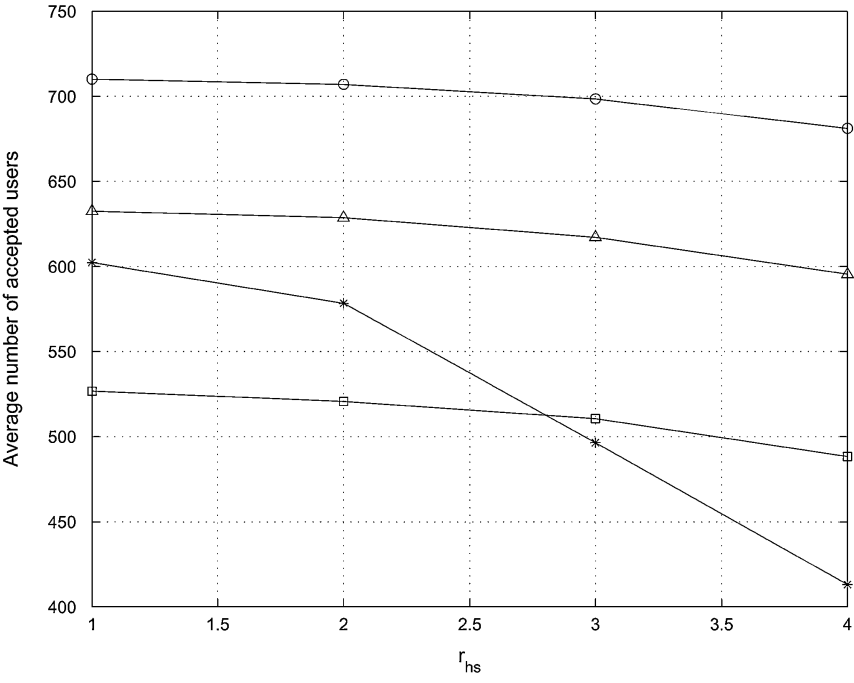

(a) $12-\mathrm{kb} / \mathrm{s}$ and $64-\mathrm{kb} / \mathrm{s}$ services

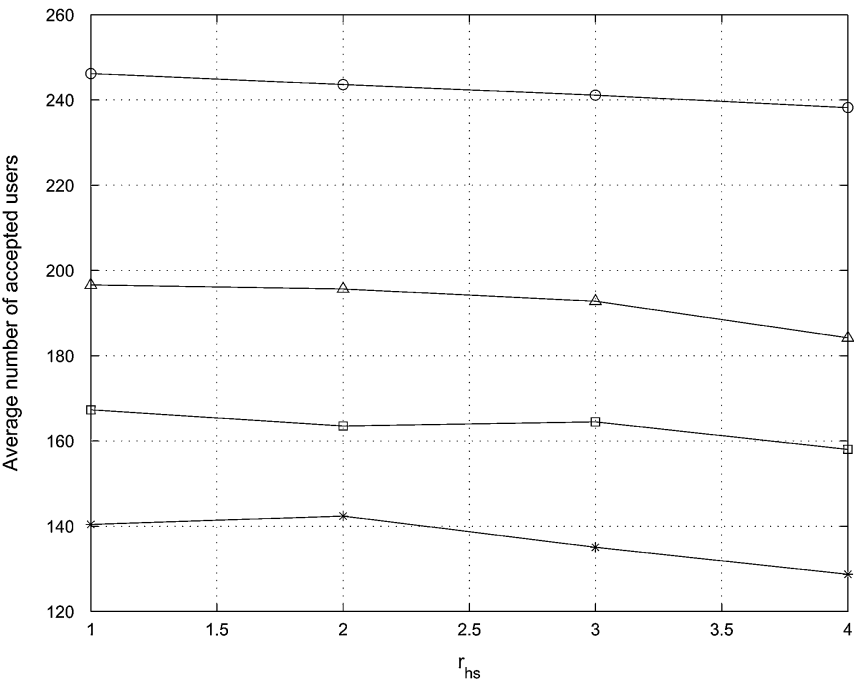

(b) $12-\mathrm{kb} / \mathrm{s}, 64-\mathrm{kb} / \mathrm{s}$ and $384-\mathrm{kb} / \mathrm{s}$ services

$*$ Minimum attenuation

- Optimum with perfect estimation

$\triangle$ Optimum with nominal SIRs and $\sigma_{\alpha}=1.5 \mathrm{~dB}$

$\square$ Optimum with nominal SIRs and $\sigma_{\alpha}=3 \mathrm{~dB}$

Fig. 6. Average number of accepted users.

SIRs (or SSIRs). In this section, capacity is evaluated by means of simulations, and the effect of estimation errors is investigated.

The simulation setting corresponds to that described in Section VI-B with $M=36$, except for the following differences. First, wraparound is introduced in order to avoid edge effects. Second, two different service combinations are considered: one with $50 \% 12-\mathrm{kb} / \mathrm{s}$ and $50 \%$ 64-kb/s users, and the other with $50 \% 12-\mathrm{kb} / \mathrm{s}, 25 \% 64-\mathrm{kb} / \mathrm{s}$, and $25 \%$ 384-kb/s users; with mean required $E_{\mathrm{B}} / N_{0}$ equal to $6 \mathrm{~dB}$ for all services. Lastly, in order to observe the effect of nonuniform traffic, a hot-spot square area extending from 3 to $4 \mathrm{~km}$ in horizontal and vertical coordinates is introduced. The parameter $r_{\mathrm{hs}}$ defines the ratio of user densities inside and outside the referred area, $r_{\mathrm{hs}}=1$ meaning uniform traffic. 


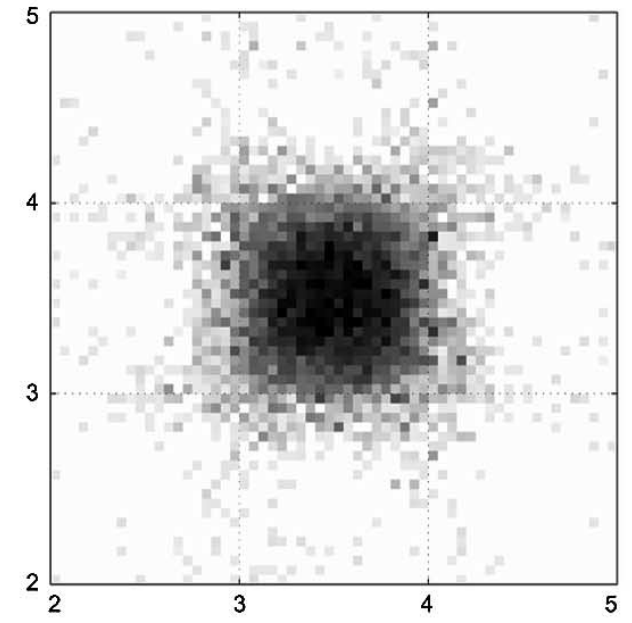

(a) Minimum attenuation; $r_{\mathrm{hs}}=3$; voice and $64 \mathrm{~kb} / \mathrm{s}$ services

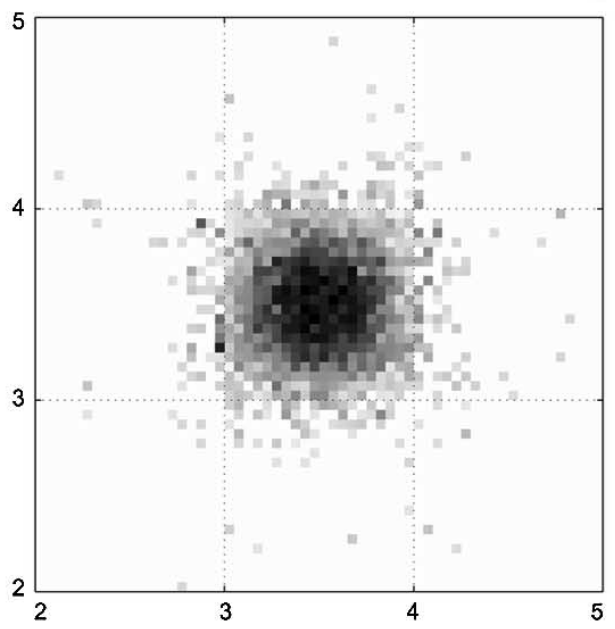

(c) Optimum with nominal SIRs and $\sigma_{\alpha}=1.5 \mathrm{~dB} ; r_{\mathrm{hs}}=3$; voice and $64 \mathrm{~kb} / \mathrm{s}$ services

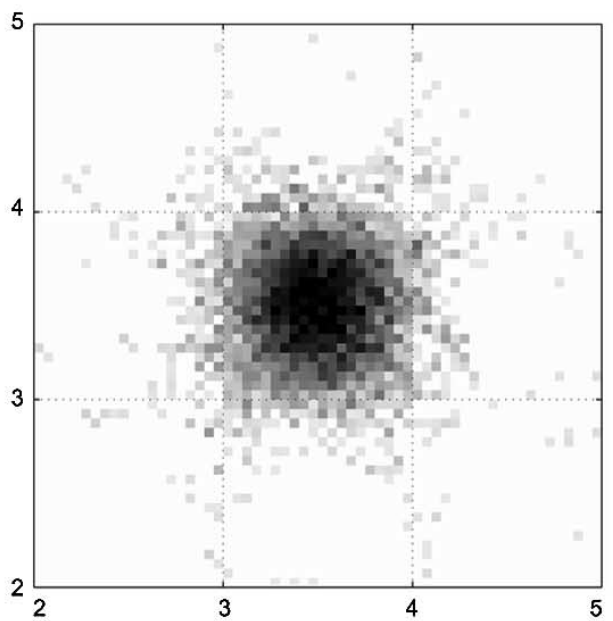

(e) Optimum with nominal SIRs and $\sigma_{\alpha}=1.5 \mathrm{~dB} ; r_{\mathrm{hs}}=3$; voice, 64 and $384-\mathrm{kb} / \mathrm{s}$ services

Fig. 7. Probability of users being assigned to hot-spot cell.

In order to assess the impact of estimation errors, the following situations are compared: an ideal one in which the algorithm operates on exact attenuations and target SIRs, and one with imperfect estimation. In the latter, we assume that no attempt is made to estimate target SIRs; instead, a "nominal"

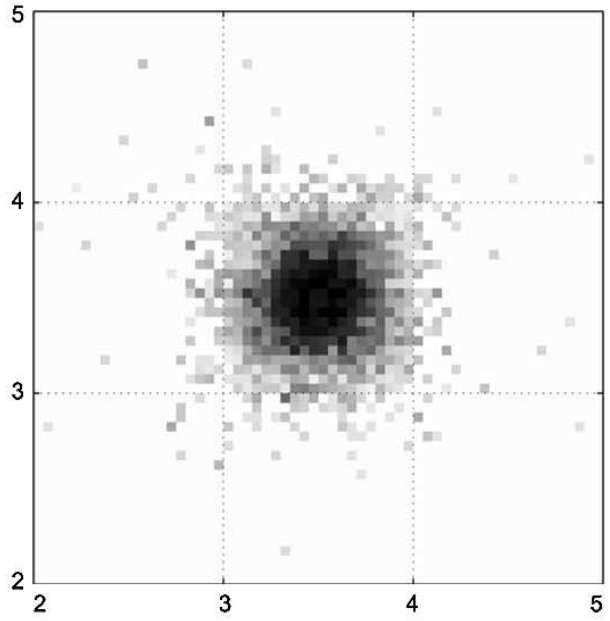

(b) Optimum with perfect estimation; $r_{\mathrm{hs}}=3$; voice and $64-\mathrm{kb} / \mathrm{s}$ services

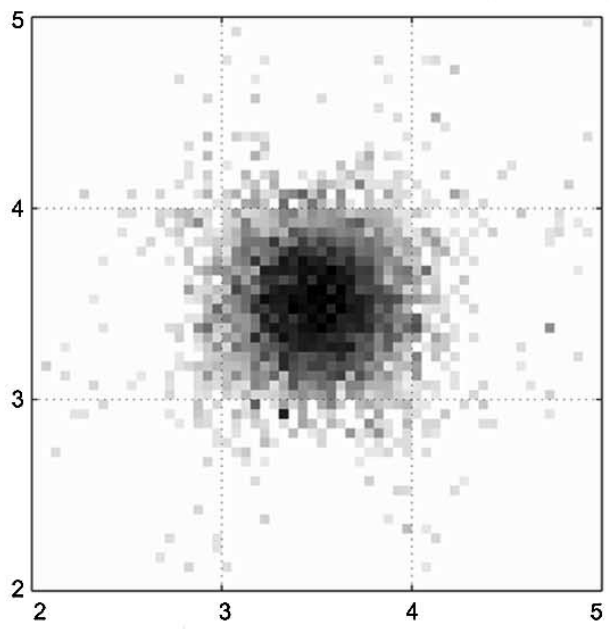

(d) Optimum with nominal SIRs and $\sigma_{\alpha}=3 \mathrm{~dB} ; r_{\mathrm{hs}}=3$; voice and $64 \mathrm{~kb} / \mathrm{s}$ services

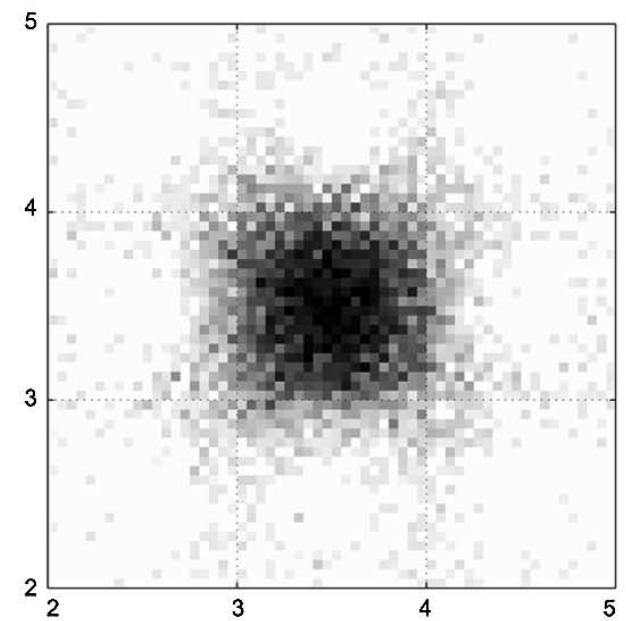

(f) Optimum with nominal SIRs and $\sigma_{\alpha}=1.5 \mathrm{~dB} ; r_{\mathrm{hs}}=1$; voice and 64-kb/s services

value is used, corresponding to the mean target value for each service class. ${ }^{6}$ In practice, these values can be obtained aver-

${ }^{6}$ Better results could be obtained by using the actual target value in the current cell as an estimate for other cells. This is because mobile speed introduces some correlation among target SIRs in different cells. 
aging actual target SIR values set by the outer loop. Estimation errors in the attenuations are modeled as independent zero-mean Gaussian random variables with standard deviation $\sigma_{\alpha}$. Background noise power is assumed to be known.

For each set of parameters, a simulation is performed consisting of a number of snapshots. In each snapshot, users are randomly generated and added one by one until infeasibility is reached. Capacity is defined as the average number of accepted users. In what follows, 200 independent snapshots are generated in the two-service case, and 400 in the three-service case (the difference is motivated by the lower number of users in the latter).

Fig. 6(a) shows the average number of accepted users in the two-service case. It is observed that the optimum assignment with perfect estimation yields a significant capacity increase with respect to minimum-attenuation assignment, especially under nonuniform traffic. It is seen that the optimum assignment is very robust to such nonuniformity, whereas in the minimum-attenuation case, the capacity falls rapidly with increasing $r_{\text {hs }}$. With imperfect estimation, the proposed algorithm still gives a capacity increase, except for uniform traffic and large estimation errors. It is remarkable that the degradation of the optimum assignment caused by estimation errors is approximately independent of $r_{\mathrm{hs}}$. The results for the three-service traffic mix are shown in Fig. 6(b). In this case, a similar behavior is observed, but the capacity increase is higher. This is due to the coarser spatial granularity caused by the presence of 384-kb/s users. Here, even with uniform traffic and large estimation errors, an important capacity increase is observed.

Fig. 7 illustrates the effect of the algorithm in terms of cell breathing. The simulation area is divided into square 50-m-size pixels. In each pixel, the probability of a user being assigned to the base station located in the center of the hot-spot area, at coordinates $(3.5,3.5)$, is computed and plotted as a grey level. The resulting graphs give a measure of the hot-spot cell area. Cell shrinking as a function of the assignment method for nonuniform traffic can be appreciated in Fig. 7(a)-(d). The optimum algorithm reduces the size of the hot-spot cell as compared to the minimum-attenuation criterion, the reduction being more important for lower estimation errors. The cell-breathing effect, as a result of traffic distribution, is recognized in Fig. 7(f) and (c), which correspond to the optimum algorithm with moderate estimation errors in the two-service case. Lastly, a comparison of Fig. 7(c) and (e) reveals that the variation in cell size is slightly lower in the three-service case, due to the spatial granularity.

\section{APPLICATIONS OF THE ALGORITHM}

The proposed algorithm shows that the optimum assignment for the uplink of a CDMA network can be found in a finite number of steps with polynomial complexity. Apart from the theoretical significance of this result, the algorithm can be used to determine the optimum assignment and power values in situations where centralized information is available and the system parameters can be assumed to be fixed (compared with the time scale of the algorithm).
In practical applications, where system conditions vary dynamically, the optimum assignment has to be updated accordingly. One approach would be to apply the algorithm whenever a change occurs in the system. However, such variations can be dealt with more efficiently as follows. If a new user arrives, the entry algorithm described in Section V-B can be applied. If one user leaves, the optimum assignment for the remaining users can be obtained by means of an "exit" procedure, which is defined as the reverse of the entry procedure. If one or several users change their conditions (attenuations or target SSIRs), each of them requires an exit procedure followed by an entry with the new parameters. In this way, variations in the system conditions can be tracked without continuously resorting to the whole algorithm; obviously, periodical "resetting" is desirable to prevent error accumulation.

In a real system, due to the centralized feature of the algorithm, the relevant information must be carried to a central node and updated whenever users arrive at or leave the system, change their target SSIRs, or experience variations in path losses. The amount of information to be transmitted, however, is rather small, as we now discuss. Path losses can be adequately represented using 7 bits with $1-\mathrm{dB}$ quantization. If each user measures and transmits attenuation values from seven cells every $0.1 \mathrm{~s}, \mathrm{a} 500-\mathrm{b} / \mathrm{s}$ uplink signaling channel is enough. This rate can be reduced by source coding, or using nonperiodical, event-driven transmission. For a cell with no more than 50 users, the attenuation information amounts to $25 \mathrm{~kb} / \mathrm{s}$ at most. Nominal SIR or $E_{\mathrm{B}} / N_{0}$ values can presumably be coded with six bits or less, and need to be updated only at bearer service changes, which can be assumed to happen no more than once per second per user as an average. This gives an additional rate of $300 \mathrm{~b} / \mathrm{s}$ per cell. Thus, the amount of signaling traffic required by the algorithm is not impractical.

It should be noted that the algorithm calculates a solution; commands then have to be sent to the mobiles in order to set the computed assignment and powers. The latter can alternatively be achieved by a combination of open and closed loops, as in current systems.

\section{CONCLUSION}

A centralized algorithm has been proposed that finds the optimum base-station assignment for the uplink of a DS-CDMA cellular network and its associated powers, allowing transmission power constraints. The algorithm features polynomial complexity in the numbers of users and base stations. Capacity has been evaluated in a practical setting with estimation errors, and it has been shown that the proposed algorithm can provide a significant capacity increase, compared with minimum-attenuation assignment, especially for nonuniform traffic.

\section{APPENDIX \\ PROOFS OF PROPOSITIONS}

Proof of Proposition 1: See [10].

Proof of Proposition 2: The proof given in [2] is easily extended to our setting.

Proof of Proposition 3: The result immediately follows from (9)-(11). 
Proof of Proposition 4: As a consequence of Proposition 2 , for any user $i$, the optimum assignment is that which minimizes $P(i)$. Furthermore, the slope of each line in the power diagram depends on the assignment. This establishes the first part of the proposition. Since the intersection point is common for all users $b$, the stated equality of SSIRs follows.

Proof of Proposition 5: It is sufficient to consider $u=$ $t+1$ and $\partial \Lambda_{k, t}(m, n ; p) / \partial p>0$. If transition $t+1$ includes the change of a mobile $i$ from cell $m$ to $n$ (i.e., it is a single transition of this user or a multiple transition that includes this), $\Gamma_{k, t}(i, n ; p)$ is an increasing function of $p$ with $\Gamma_{k, t}(i, n ; p)<$ $\gamma(i, n)$ for $p<p_{k, t+1}$, and

$$
\begin{aligned}
\Gamma_{k, t}\left(i, n ; p_{k, t+1}\right) & =\frac{\left(r_{k, t}(m)+s_{k, t}(m) p_{k, t+1}\right) \gamma(i, m) \alpha(i, n)}{\left(r_{k, t}(n)+s_{k, t}(n) p_{k, t+1}\right) \alpha(i, m)} \\
& =\gamma(i, n) .
\end{aligned}
$$

Imagine that in segment $t$, before the transition takes place (i.e., with $\left.p<p_{k, t+1}\right)$, user $i$ is switched to cell $n$, maintaining the power values. By executing this switching at $p$ arbitrarily close to $p_{k, t+1}$, feasibility of the new assignment can be assured. Then user $i$ will have too low a SSIR in $n$ and a foreign $\operatorname{SSIR} \Gamma_{k, t+1}(i, m ; p)=\gamma(i, m)$ in $m$. In order to raise the former to its target value, all powers must increase (Proposition 1 ), and this raises $\Gamma_{k, t+1}(i, m ; p)$ (because switching user $i$ back to $m$ would decrease the required powers). This shows that $\Gamma_{k, t+1}(i, m ; p)>\gamma(i, m)$ for $p<p_{k, t+1}$. Hence, $\Gamma_{k, t+1}(i, m ; p)$ is a decreasing function of $p$, and consequently $\Lambda_{k, t+1}(m, n ; p)$ increases with $p$.

If transition $t+1$ does not include a change from cell $m$ to cell $n$, the reasoning in the previous paragraph can be applied, using continuity arguments, introducing an additional, fictitious user that changes from $m$ to $n$ at $p=p_{k, t+1}$, with an arbitrarily small target SSIR [10].

Proof of Proposition 6: From (9), it is seen that

$$
\frac{\partial \Lambda_{k, t}(m, n ; p)}{\partial p}=-\frac{\partial \Lambda_{k, t}(n, m ; p)}{\partial p}
$$

which establishes antisymmetry and totality. To prove the transitivity, we observe that for any $l, m, n \in\{1, \ldots, M\}$

$$
\Lambda_{k, t}(l, n ; p)=\Lambda_{k, t}(l, m ; p) \Lambda_{k, t}(m, n ; p)
$$

and hence

$$
\begin{aligned}
\frac{\partial \Lambda_{k, t}(l, n ; p)}{\partial p}=\frac{\partial \Lambda_{k, t}(l, m ; p)}{\partial p} & \Lambda_{k, t}(m, n ; p) \\
& +\frac{\partial \Lambda_{k, t}(m, n ; p)}{\partial p} \Lambda_{k, t}(l, m ; p)
\end{aligned}
$$

which is positive if $\partial \Lambda_{k, t}(l, m ; p) / \partial p$ and $\partial \Lambda_{k, t}(m, n ; p) / \partial p$ are.

\section{ACKNOWLEDGMENT}

The authors are grateful to the anonymous reviewers as well as the Editor for Spread Spectrum, Prof. G. E. Corazza, for their valuable comments and suggestions. L. Mendo also thanks Prof. R. Criado for pointing out [6].

\section{REFERENCES}

[1] S. V. Hanly, "Information Capacity of Radio Networks," Ph.D. dissertation, Cambridge Univ., Cambridge, U.K., Aug. 1993.

[2] S. V. Hanly, "An algorithm for combined cell-site selection and power control to maximize cellular spread spectrum capacity," IEEE J. Select. Areas Commun., vol. 13, pp. 1332-1340, Sept. 1995.

[3] R. D. Yates and C.-Y. Huang, "Integrated power control and base station assignment," IEEE Trans. Veh. Technol., vol. 44, pp. 638-644, Aug. 1995.

[4] R. D. Yates, "A framework for uplink power control in cellular radio systems," IEEE J. Select. Areas Commun., vol. 13, pp. 1341-1347, Sept. 1995.

[5] L. Mendo and J. M. Hernando, "On dimension reduction for the power control problem," IEEE Trans. Commun., vol. 49, pp. 243-248, Feb. 2001.

[6] G. Brassard and P. Bratley, Fundamentals of Algorithmics. Englewood Cliffs, NJ: Prentice-Hall, 1996.

[7] B. Noble and J. W. Daniel, Applied Linear Algebra, 3rd ed. Englewood Cliffs, NJ: Prentice-Hall, 1988.

[8] J. K. Truss, Discrete Mathematics for Computer Scientists. Reading, MA: Addison-Wesley, 1991.

[9] G. H. Golub and C. F. Van Loan, Matrix Computations, 2nd ed. Baltimore, MD: Johns Hopkins Univ. Press, 1989.

[10] L. Mendo, "Capacity in W-CDMA Cellular Systems," Ph.D. dissertation, Polytechnic Univ. of Madrid, Madrid, Spain, Dec. 2001. (in Spanish).

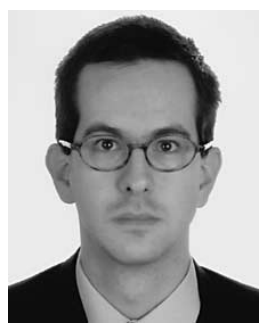

Luis Mendo was born in Madrid, Spain, on June 20, 1973. He received the M.Sc. and the Ph.D. degrees in telecommunication engineering from the Polytechnic University of Madrid, Madrid, Spain, in 1997 and 2001, respectively.

He has worked in radio network planning, and is currently with the Polytechnic University of Madrid. He is a coauthor of two textbooks on CDMA cellular systems, and has published several papers in the fields of CDMA system capacity and power control. His current research interests include CDMA networks, teletraffic, and Monte Carlo methods.

Dr. Mendo is a recipient of a national prize from the Telecommunication Engineering Professional Organization for his Ph.D. dissertaiton, and of two national awards for his M.Sc. thesis.

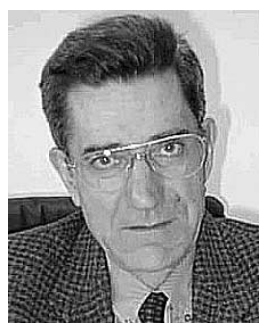

José M. Hernando (M’94) received the M.S. degree in communications engineering and the Ph.D. degree from Madrid Polytechnic University, Madrid, Spain, in 1967 and 1970, respectively.

Between 1967-1970, he was with ITT Laboratories of Spain doing research work in teletraffic theory. From 1970 until 1977, he was a Senior Engineer in the Communications Department of Iberia Airlines, Spain, working on planning, implementation, and maintenance of mobile radio networks, ground and air-to-ground in $\mathrm{HF}$ and VHF/UHF for both voice and data radiocommunications. In 1977, he joined the Signals Systems and Radiocommunications Department, Madrid Polytechnic University. Since then, he has been a Senior Professor devoted to educational and research work in radiocommunications. He has served as the Spanish Delegate in conferences and working groups in the Radiocommunications Sector of the International Telecommunications Union.

Dr. Hernando is member of the Spanish Bar of Telecommunications Engineers. 